\title{
Comparative Analysis of Friction in Vibration-Driven System and Its Dynamic Behaviors
}

\author{
Sainan Wang, Xiong Zhan and Jian Xu* \\ School of Aerospace Engineering and Applied Mechanics, Tongji Univerisity, Shanghai, People's Republic of \\ China \\ Email: xujian@tongji.edu.cn
}

\begin{abstract}
Numerical simulations and experimental investigations of single module vibration-driven system with friciton have been stuidied in this paper. Friction models can be divided into two classes, namely static and dynamic models. Thus, we just compare the anisotropic Coulomb friction and that of the LuGre model. Through numerical simulations, one can find the LuGre model ha has a good agreement with the experimental data from both qualitative and quantitative viewpoints. Moreover, through changing the physical parameters of the system, the vibration-driven system with the LuGre model can dispaly lots of dynamic behaviors expect for the four types movement forms, such as quasi-periodic motion and periodic two motion.
\end{abstract}

Keywords: Coulomb friction model, LuGre model, vibration-driven system, stick-slip, dynamic behavior.

\section{Introduction}

Vibration-driven robots have advantages over other vehicles such as legged, wheeled and propelled robots. They are simple in design, and can be fabricated into small sizes. The micro robots can be used to detect the narrow pipes, rescue the life in disaster and inspect the human cardiovascular, etc. The vibration-driven system can move rectilinearly owing to the periodic motion of the internal mass and the resistances between the whole system and the environment. In the recent years, these systems have attracted lots of scholars' attention.

Russia's academy of science, F.L Chernous'ko was the first one to study the dynamic systems with movable internal mass. In 2005, F.L Chernous'ko studied a single module vibration-driven system in anisotropic Coulomb friction medium [1]. From then on, many scholars studied these systems from different viewpoints. Until now, according to the number of module, the system can be divided into single module [2-11], two-module [12,13] and multi-module [14-15]. Therein, they discussed the vibration-driven system under different driving force, like velocity control mode (two-phase motion) [5], acceleration control mode (three-phase motion) [7, 9, 12] and harmonic excitation [3]. In [12, 13], the authors analyzed a single driven-vibration system with two internal masses. In 2013, Hongbin Fang and Jian Xu [8] discussed the single module vibration-driven system with the Coulomb dry friction in terms of sliding bifurcation, they found that these sliding bifurcations organized different types of transitions between slip and sticking motions in the system. In this paper, by simulation and experiments data, we can find four types of movement forms of the single module vibration-driven system, i.e., three types of stick-slip motion and one type of non stick-slip motion.

Friction is a complicated phenomenon in mechanical systems. Several friction effects have been found, such as Coulomb friction effect, viscous friction effect, Stribeck effect, time lag effect, etc. The magnitude of friction force between the contacting surfaces depends on several factors, such as the relative velocity of the surfaces, the normal load, the interface material, the lubrication condition and time [16, 17]. Until now, there are tens of kinds of friction models [18-20]. According to whether the mathematical expressions of friction models can be written in the differential form or not, the friction models can be divided into two classes, i.e., dynamic and static models. The simplest fiction model Coulomb friction belongs to static model. Examples of dynamic models are the Bristle model, the Dahl model and the LuGre model. The 
dynamic friction model can describe many friction effects that the static model cannot [21-24]. Some scholars modified these dynamic models to make the models have a great agreement with experiment data [25-25]. One should notice the identification of the dynamic models, which is still a difficultly. In the present study of vibration-driven system, the scholars just use the Coulomb friction $[1,3,-5,9,10,14]$, the viscous friction $[7,10,12,28]$ or the quadratic friction [10], they never consider the dynamic friction model. In this paper, the single module of vibration-driven system with LuGre model will be discussed.

The rest of the paper is organized as follows: Section 2 describes the single module of vibration-driven system and anisotropic friction models in detail. In Section 3, we will describe the experiment settings and measurement set-up. In Section 4, the simulations of the vibration-driven system with Coulomb friction and LuGre model will be compared with experimental data. Many dynamic behaviours of the vibration-driven system with LuGre model will be presented in the Section 5 . The paper is concluded in Section 6

\section{Single Module of Vibration-driven System and Friction}

\subsection{Mechanical Model}

A mass $m$ vibrates periodically relative to an absolutely rigid body $M$, which can move on a horizontal plane due to the interaction with mass $m$ and the anisotropic friction between the body $M$ and the plane. To describe the motion of the vibration-driven system, the absolute displacement of the body $M$ is denoted by $x$, and the displacement of the mass $m$ relative to the body $M$ by $\xi$. Then the corresponding velocity and acceleration of the body $M$ and mass $m$ is $\dot{x}, \ddot{x}$ and $\dot{\xi}, \ddot{\xi}$, respectively.

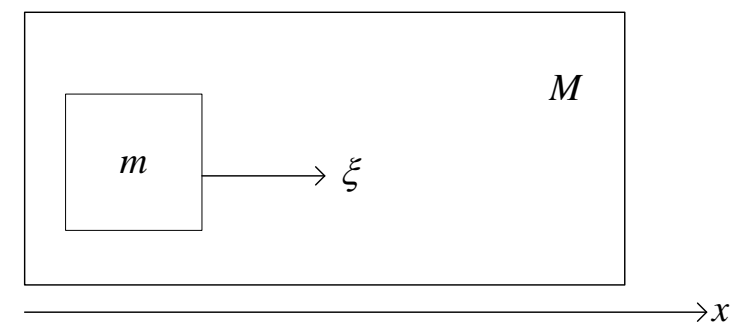

Figure 1. Mechanical model.

The equation of the whole system is governed by

$$
\left\{\begin{array}{l}
M \ddot{x}=F-R, \\
m(\ddot{x}+\ddot{\xi})=-F,
\end{array}\right.
$$

where $F$ is the interaction force between the body $M$ and mass $m$. Harmonic excitation is chosen as

$$
\xi(t)=A \sin (\omega t)
$$

The friction force between the body $M$ and the plane is $R$. Specific forms of friction $R$ of Coulomb friction and that of LuGre model will be introduced in the next section.

Substituting (2.2) into (2.1) and eliminating the force $F$, we obtain

$$
\ddot{x}=A \mu \omega^{2} \sin (\omega t)-\frac{R}{M+m}
$$

Where $\mu=\frac{m}{M+m} \in(0,1)$. For the significance of anisotropic friction force $R$ in the vibration-driven system, we consider two friction models in detail, namely, the Coulomb friction and the LuGre model. 


\section{$2.2 \quad$ Two Friction Models}

According to whether the mathematical expressions of friction models can be written in the differential form or not, the friction models can be divided into two classes, i.e., dynamic and static models. Static friction model can describe a friction phenomenon as a function of the relative velocity of the contacted surfaces, for example, the Coulomb friction model which is the relative simple friction model in form. The dynamic friction models contain bristle model, Dahl model and LuGre model. However, to simulate the dynamic behavior of systems with friction of bristle model, very tiny step size and long simulation time are needed. Besides, the simulation performance is generally not good enough [19]. For the Dahl model, friction phenomenon of stick-slip and Stribeck cannot be simulated [25]. Thus, in the paper we just study the dynamic system with friction of the LuGre ,one among the dynamic modes.

\subsubsection{The Coulomb Friction Model}

The mathematical expression of Coulomb friction model is defined as follows

$$
R=\left\{\begin{array}{cc}
\mu_{+}(M+m) g & \text { if } v>0, \\
-\mu_{-}(M+m) g & \text { if } v<0, \\
\mu_{+}(M+m) g & \text { if } v=0 \text { and } f_{e}>\mu_{+}(M+m) g, \\
f_{e} & \text { if } v=0 \text { and }-\mu_{-}(M+m) g \leq f_{e} \leq \mu_{+}(M+m) g, \\
-\mu_{-}(M+m) g & \text { otherwise. }
\end{array}\right.
$$

Here, $f_{e}$ is the resultant of all forces, except for the Coulomb friction that body $M$ subjected to. $g$ is acceleration of gravity. $\mu_{+}$and $\mu_{-}$is the forward and backward dynamic friction coefficients, respectively. For $\mu_{+} \neq \mu_{-}$, the friction is anisotropic. In this case, the anisotropic friction will make the whole system have a net periodic displacement.

\subsubsection{LuGre Model}

LuGre model is an extension of the Dahl model. Furthermore, it can capture the Stribeck effect and describe stick-slip effect, which Dahl model cannot. When coupled to the single degree of freedom system shown in Fig.1, the governed equation of the vibration-driven system becomes a three dimensional first-order differential equation. The anisotropic LuGre model is constructed as follows

$$
\dot{z}=\left\{\begin{array}{l}
\dot{x}-\frac{\sigma_{0} z+\sigma_{1} \dot{z}+\sigma_{2} \dot{x} z}{(M+m) g\left(\mu_{+}+\left(f_{+}-\mu_{+}\right) e^{\left.-\left(\frac{\dot{x}}{v_{s}}\right)^{2}\right)}\right.} \text { if } \dot{x}>0, \\
\dot{x}+\frac{\sigma_{0} \dot{x} z}{(M+m) g\left(\mu_{-}+\left(f_{-}-\mu_{-}\right) e^{\left.-\left(\frac{\dot{x}}{v_{s}}\right)^{2}\right)}\right.} \text { if } \dot{x}<0 .
\end{array}\right.
$$

where $\sigma_{0}$ is the stiffness of the bristle, $\sigma_{1}$ is micro damping coefficient, $\sigma_{2}$ is viscous friction coefficient and $v_{s}$ is Stribeck velocity which is empirical.

Substituting (2.6) into (2.5), and we can find out that the friction force $R$ is a function of $z$ and $\dot{x}$.

$z$ denotes an internal state variable standing for the average deformation of the bristle.

Until now, the excitation force and friction models are known. In the next section, we will introduce the experiment settings briefly. 


\section{The Experiments Settings}

In this part, we will present the experiment design and setup. Based on the above section, the driving force is sinusoidal and the friction force is anisotropic. Now, the following experiment design can meet both of the conditions.

\subsection{The Driving Force}

Fig. 2 shows the prototype of the vibration-driven system. The prototype consists of three main parts: the spring steel beam, the piezoelectric patches and the lumped mass. The two piezoelectric patches are attached to the top of the spring steel beam. When one of them stretches and the other one contracts, it equals to imposing a torque on the spring steel beam at the both top and bottom ends of the two piezoelectric patches. The internal mass $m$ is implemented by the lump mass at the end of spring steel beam. A voltage whose amplitude is in the range of 10 to $150 \mathrm{~V}$ is applied to the piezoelectric patches, which allows to excite the cantilever beam in a frequency below $5000 \mathrm{HZ}$. Under the actuation of the piezoelectric patches, the beam vibrates and then the lumped mass vibrates sinusoidally. Due to the small amplitude of the bottom mass, we can omit the vertical movement of the bottom mass, thus the lumped mass just makes the horizontal motion. The cantilever beam actuated by piezoelectric patches is controlled by the following equation

$$
E I \frac{\partial^{4} u}{\partial x^{4}}+c \frac{\partial u}{\partial t}+\rho A_{1} \frac{\partial^{2} u}{\partial x^{2}}=2 \frac{\partial}{\partial x}\left[T(t)\left(\delta(x-0)+\delta\left(x-l_{0}\right)\right)\right], 0<x<L .
$$

where $E I$ is flexural rigidity, $c, \rho$ and $A_{1}$ is damping, linear density and cross-section area of the spring steel beam, respectively. $T(t)$ and $l_{0}$ denote bending moment and length of the piezoelectric patches. $L$ is the length of the beam.

The bending moment of the piezoelectric patches imposed on the beam [32] is

$$
T(t)=d_{31}\left(h_{1}+h_{2}\right) b E_{2} V(t) .
$$

Here, $d_{31}$ is piezoelectric constant, $b$ is the width of the piezoelectric patches, $E_{2}$ is electric field intensity, and $V(t)$ is voltage. $h_{1}, h_{2}$ is shown in Fig. 2.

Using the method of separation of variables,

$$
u(x, t)=\sum_{i=1}^{\infty} Y_{i}(x) \eta_{i}(t)
$$

where $Y_{i}(x)$ is the normal modal and $\eta_{i}(t)$ is the normal coordinate. For the vibration of the beam, $Y_{i}(x)$ owns the following form:

$$
Y_{i}(x)=C_{i 1} \cos \beta_{i} x+C_{i 2} \sin \beta_{i} x+C_{i 3} \cosh \beta_{i} x+C_{i 4} \sinh \beta_{i} x,
$$

where $\beta_{i}^{4}=\frac{\rho A \omega_{i}^{2}}{E I}, i=1,2, \cdots$, the boundary conditions of the beam are

$$
\begin{array}{ll}
x=0: & Y(0)=0, Y^{\prime}(0)=0 ; \\
x=L: & Y^{\prime \prime}(L)=0, E I Y^{\prime \prime \prime}(L)=-\omega^{2} m_{0} Y(L) .
\end{array}
$$

Solving (3.1)-(3.4) yields

$$
\begin{aligned}
& u(x, t)=\sum_{i=1}^{N} C_{i}\left[\cos \beta_{i} x-\cosh \beta_{i} x+r_{i}\left(\sin \beta_{i} x-\sinh \beta_{i} x\right)\right] \\
& \cdot\left(B_{i} e^{-\zeta \omega_{i} t}\left[\sin \varphi \cos \omega_{d} t+\frac{\omega_{i}}{\omega_{d}}\left(\zeta_{i} \sin \varphi-\frac{\omega}{\omega_{i}} \cos \varphi\right) \sin \omega_{d} t\right]+B_{i} \sin (\omega t-\varphi)\right)
\end{aligned}
$$


The steady solution is

$$
u(x, t)=\sum_{i=1}^{N} C_{i}\left[\cos \beta_{i} x-\cosh \beta_{i} x+r_{i}\left(\sin \beta_{i} x-\sinh \beta_{i} x\right)\right] B_{i} \sin (\omega t-\varphi) .
$$

According to(3.7), it is easy to find that the vibration of the beam is sinusoidal. That is to say $\xi(t)=u(L, t)$, this condition meets the demand of the driving force given in $(2.2)$.

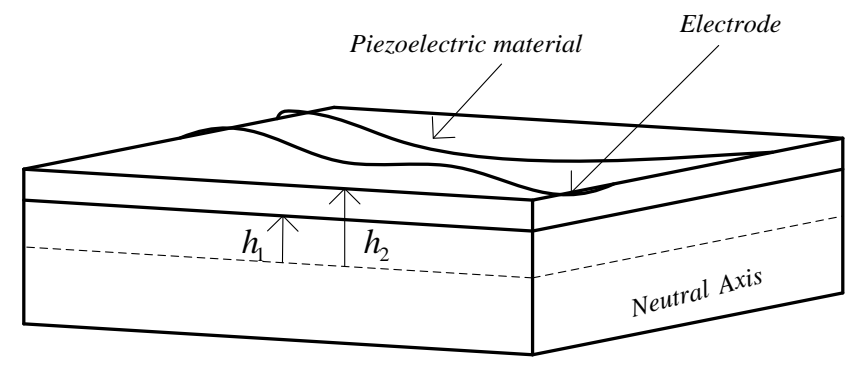

Figure 2. Piezoelectric beam.

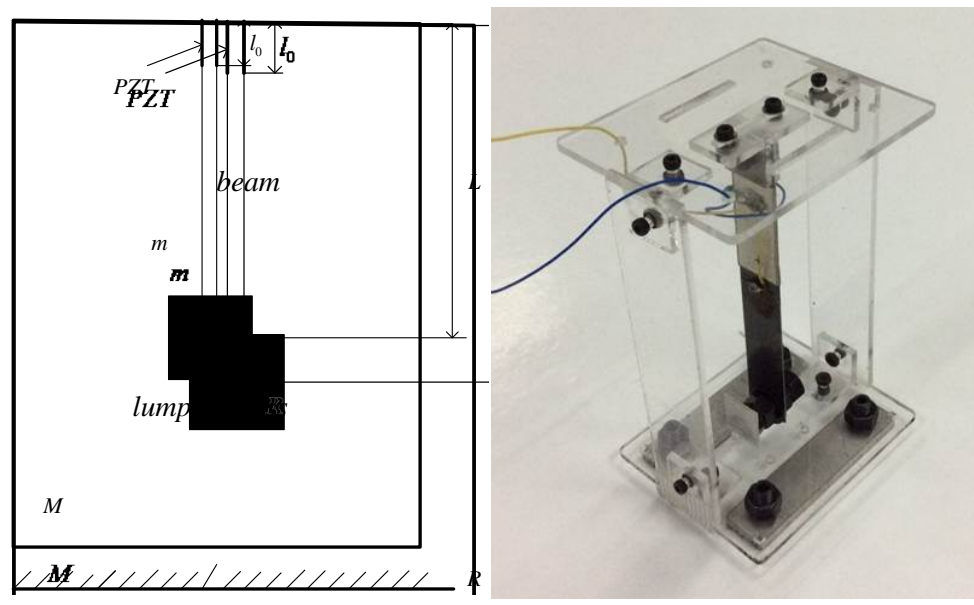

Figure 3. The experiments settings of the single module vibration-driven system.

\subsection{The Anisotropic Friction}

In order to make the whole system move forward, we construct the following experimental rig shown in Fig.4. Thus, the anisotropic friction could be expressed as:

$$
\begin{aligned}
& F_{S_{+}}=F_{S}-M g \sin \theta=\left(f_{0} \cos \theta-\sin \theta\right) M g=f_{+} M g, \\
& F_{S_{-}}=F_{S}+M g \sin \theta=\left(f_{0} \cos \theta+\sin \theta\right) M g=f_{-} M g, \\
& F_{C+}=F_{C}-M g \sin \theta=\left(\mu_{0} \cos \theta-\sin \theta\right) M g=\mu_{+} M g, \\
& F_{C-}=F_{C}+M g \sin \theta=\left(\mu_{0} \cos \theta+\sin \theta\right) M g=\mu_{-} M g .
\end{aligned}
$$

Where $f_{+}, f_{-}$and $\mu_{+}, \mu_{-}$is the forward (backward) static friction coefficient and the forward (backward) Coulomb coefficient, respectively. $F_{S_{+}}, F_{S_{-}}, F_{C_{+}}$and $F_{C_{-}}$are the corresponding friction forces. $\theta$ is the angle of the inclined track. On the inclined slope, anisotropic friction is achieved. 

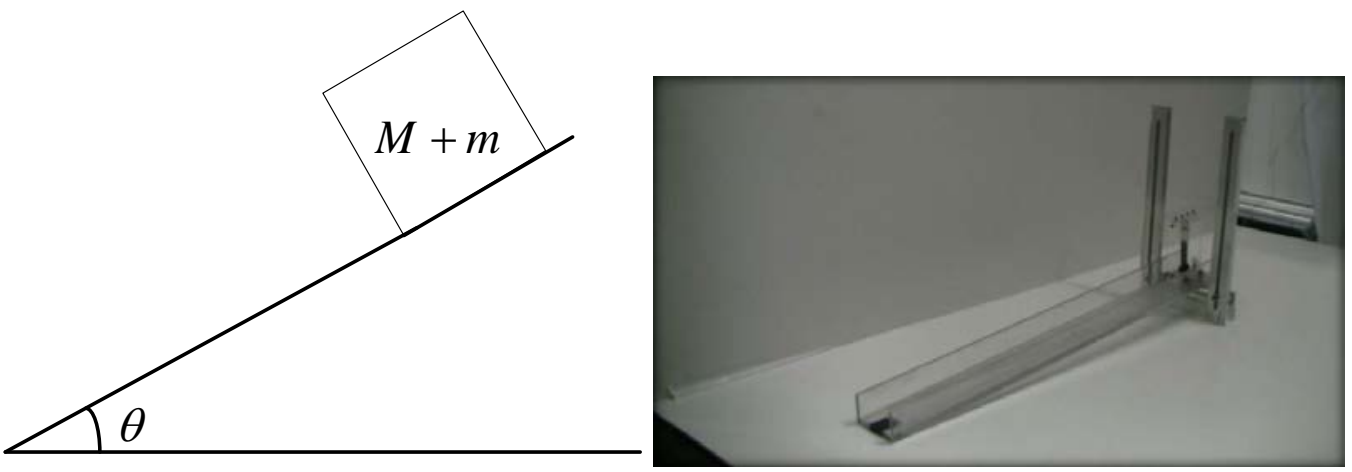

Figure 4. The inclined track.

Until now, both of the driving force and the friction force meet the theoretical requirements. In the next section, devices measuring the velocity of the system will be introduced.

\subsection{The Measurement Setup}

Fig . 5 displays the measurement setup of the experiment. The velocity of the prototype is measured by the testing equipment-Polytec scanning vibrometer (precision $0.001 \mathrm{~mm} / \mathrm{s}$ ). The signal generator outputs sinusoidal voltage signal, which is am plified by the power supply of piezoelectric ceramic and applied to the piezoelectric patches.

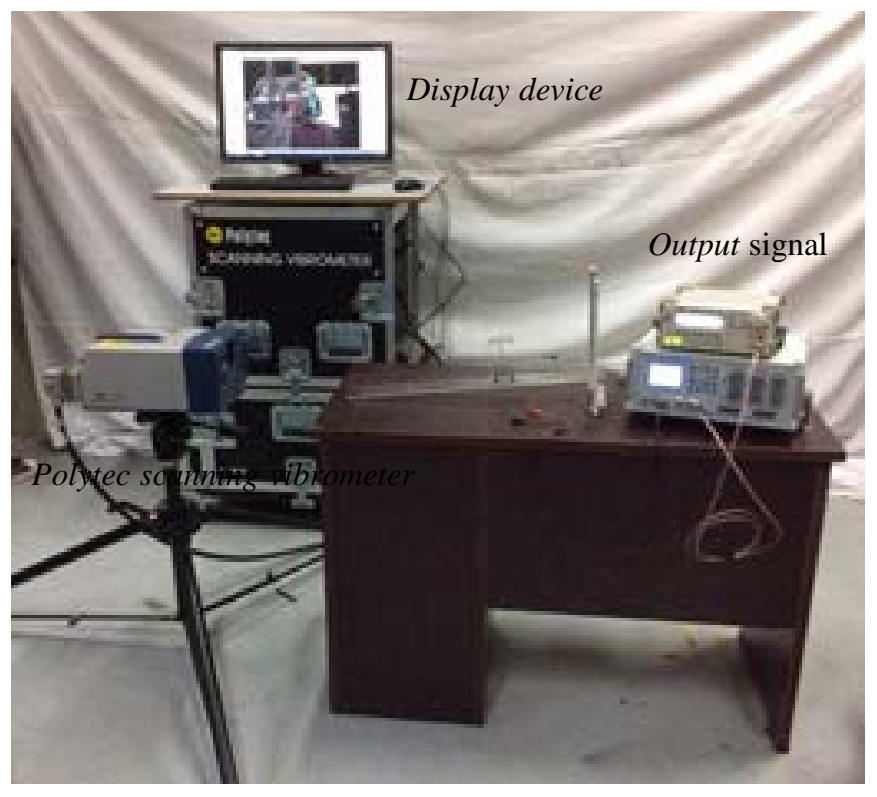

Figure 5. The measurement setup

Using the equipment-Polytec scanning vibrometer, we can obtain the absolute velocity and the convected velocity of the whole system. Moreover, the relative velocity can be obtained with the absolute velocity $\dot{x}+\dot{\xi}$ and the convected velocity $\dot{x}$. Thus, the driving force of the system is obtained.

In the next section, we simulate the dynamic behavior of the single module vibration-driven system and compare it with the experimental data. 


\section{The Comparison of the Numerical Simulation and Experimental Data}

\subsection{Four Types of Movement Forms}

In 2013, Hongbin Fang [9] talked about the single module vibration-driven system with Coulomb dry friction in terms of sliding bifurcation. He found that these sliding bifurcations organized different types of transitions between slip and stick motions in the system. The vibration-driven system in this paper shows four types of motion with harmonic excitation shown in Fig.5. The Figure is plotted by using Coulomb friction. We have carried out numerical simulations of equations (2.3) through the $4^{\text {th }}$ order Runge-Kutt a method.

For the brevity to identify, we name the four types of motions in the following ways. From Fig. 6, type four is exactly different from the other ty pes. It is a non-stick-slip motion. If the stick time internal has two parts in a period, we call it ty pe one. If the time history of the velocity of the system is always nonnegative, we name it type two.

In order to verify which friction model is in a better agreement with the experimental data. Coulomb model and LuGre model of the friction $R$ are utilized to simulate the dynamic behaviors of the single module vibration-driven system. In the past researches, many scholars use Coulomb friction, linear viscous friction and quadratic friction to do the numerical simulation of the vibration-driven system. Because of the complexity of the actual circumstance, the experimental data may not have a good agreement with the numerical results.
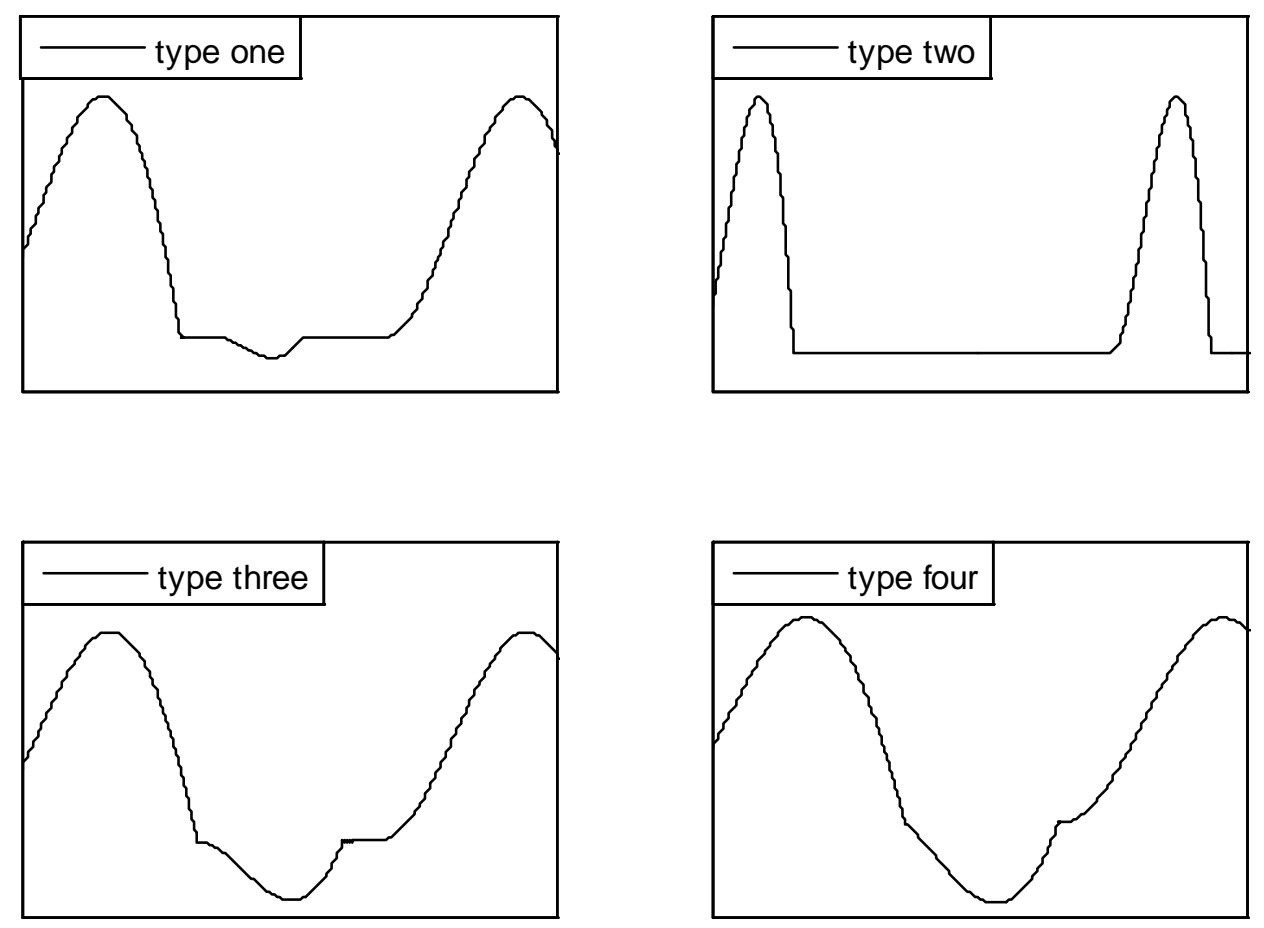

Figure 6. Four types of the vibration-driven system with the Coulomb friction.

Values of the physical parameters are as follows

$$
m=9.48 \mathrm{~g}, M=62.59 \mathrm{~g}, \omega=65.4 \pi, \mu=\frac{m}{M+m}=0.1315
$$

The friction coefficients in the experiment are $\mu_{0}=0.18, f_{0}=0.21$. Six groups of experiments of different friction coefficients are conducted to study dynamic behaviors of the single module 
vibration-driven system. The anisotropy of the friction coefficients can be implemented by adjusting the angle of the inclined plane shown in Fig. 4. From (3.8), the relationship between the angle of the slope and the backward and forward friction coefficients are

$$
\mu_{+}=\mu_{0} \cos \theta-\sin \theta, \mu_{-}=\mu_{0} \cos \theta+\sin \theta, f_{+}=f_{0} \cos \theta-\sin \theta, f_{-}=f_{0} \cos \theta+\sin \theta
$$

\subsection{The Numerical Simulation of Two Friction Models}

In the following content, dynamic behaviors of vibration-driven system with friction of the two different models are compared both qualitatively and quantitatively. From the point of quantitative analysis, we compare the mean steady-state velocity of the experiment results and numerical simulation of the vibration-driven system with the two friction models.

We should notice that in the following simulations the parameters of the LuGre model are selected as follows

$$
\sigma_{0}=4 \times 10^{5} \mathrm{~N} / \mathrm{m}, \sigma_{1}=100 \mathrm{Ns} / \mathrm{m}, \sigma_{2}=0.04 \mathrm{Ns} / \mathrm{m}, v_{s}=2 \times 10^{-3} \mathrm{~m} / \mathrm{s}
$$

\subsubsection{The First Type of Movement Forms}

The simulation is implemented under the condition that the angle of the slope is $\theta=1.7^{\circ}$.

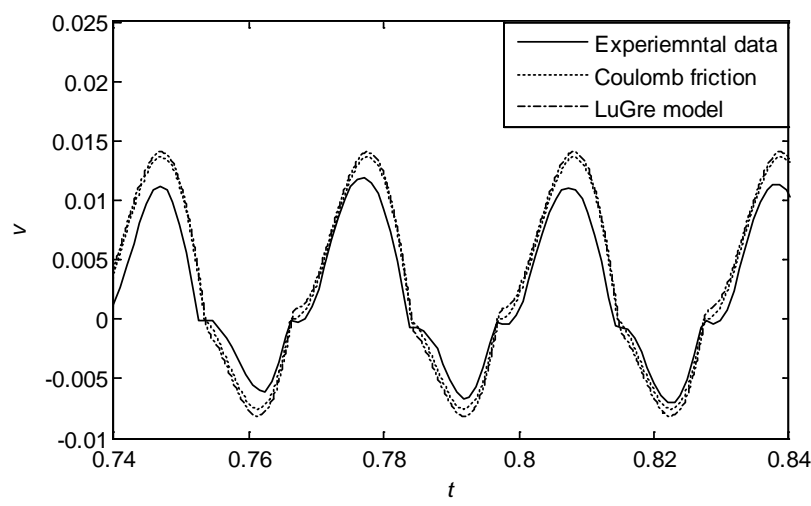

Figure 7. The time history of the dimension velocity $v$ resulting from numerical simulation of Coulomb friction in dotted curve and LuGre model in dashed curve of the exact equation of the motion and the experimental data in solid curve.

Firstly, we will give a definition of mean steady state velocity $\bar{v}$ of the rigid body $M$, $\bar{v}=\frac{x\left(t_{0}+p T\right)-x\left(t_{0}\right)}{p T}$, where $T=\frac{2 \pi}{\omega}$ is the periodic of the harmonic excitation, $x$ is the displacement

of the rigid body, $t_{0}$ is a time point meets $0 \leq t_{0} \leq T . p$ is an arbitrary integer.

From Fig.7, the simulation results of the system with Coulomb friction model have two parts of stick time internals. In the sticking parts, the velocity of body $M$ is nearly zero (neglect the computational errors). However, the experiment results and simulation with LuGre model just show two small curves.

Through simulation, one can know that the average velocity of the experimental data is about $0.0017 \mathrm{~m} / \mathrm{s}$. The mean velocity of the numerical simulation with the Coulomb friction roughly equals to $0.0020 \mathrm{~m} / \mathrm{s}$. The result of the LuGre model is about $0.0018 \mathrm{~m} / \mathrm{s}$. The simulation result of the LuGre model is closer to the experimental data. Moreover, the qualitative feature of the LuGre model is more 
consistent with the experimental data.

\subsubsection{The Second Type of Movement Forms}

The simulation is implemented under the condition that the angle of the slope is $\theta=4.7^{\circ}$.

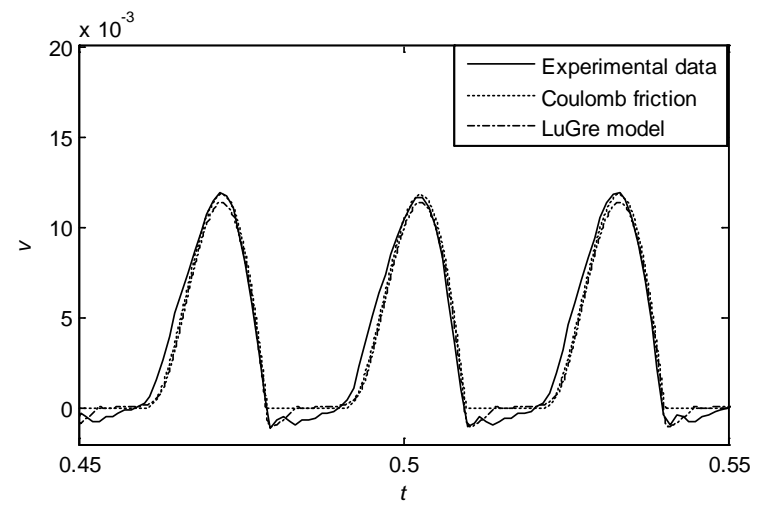

Figure 8. The time history of the dimension velocity $v$ resulting from numerical simulation of Coulomb friction in dotted curve and LuGre model in dashed curve of the exact equation of the motion and the experimental data in solid curve.

We can get the average velocity of the experiment, the simulation results with Coulomb friction and LuGre model is $0.0040 \mathrm{~m} / \mathrm{s}, 0.0040 \mathrm{~m} / \mathrm{s}, 0.0037 \mathrm{~m} / \mathrm{s}$, respectively.

When the velocity of the rigid body under the simulation of the anisotropic dry Coulomb friction is always zero, we name the corresponding time internals sticking internals.

From Fig. 8, it's easily found that, the results of both experiment and simulation with LuGre model are in good agreement. Many tiny curves can be found in the sticking internals of them two, which is apparently different from simulation with the Coulomb friction model.

\subsubsection{The Third Type of Movement Forms}

Simulation is implemented under the condition that the angle of the slope is $\theta=2.7^{\circ}$.

By simple calculation, we can obtain the average velocity of the experimental data. The numerical simulation of the differential equation with Coulomb friction and LuGre model is $0.0036 \mathrm{~m} / \mathrm{s}, 0.0038 \mathrm{~m} / \mathrm{s}, 0.0037 \mathrm{~m} / \mathrm{s}$, respectively.

To conclude, from Fig.7-Fig. 9 one may find that the stick-slip motion of the numerical simulation with the Coulomb friction is very apparent. Within sticking internals, the velocity of body $M$ remains unchanged. However, the experimental data and the numerical simulation with the LuGre model will have tiny changes around the zero velocity.

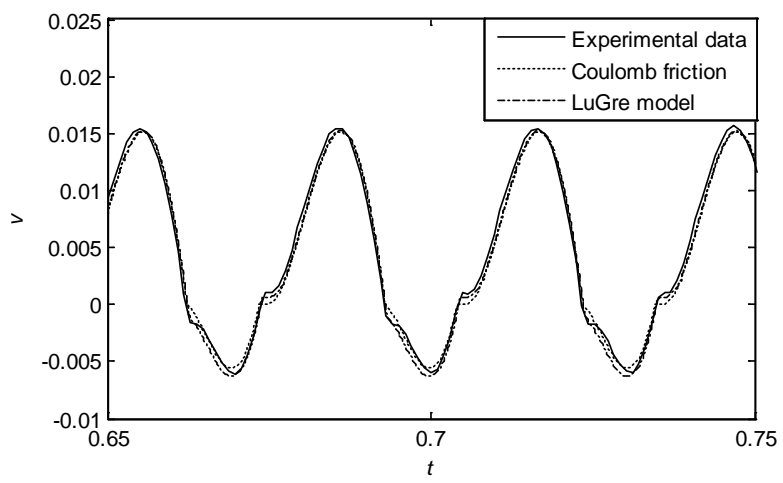

Figure 9. The time history of the dimension velocity $v$ resulting from numerical simulation of Coulomb friction in dotted curve and LuGre model in dashed curve of the exact equation of the motion and the experimental data in solid curve. 
4.2.4 The Fourth Type of Movement Forms

Simulation is implemented under the condition that the angle of the slope is $\theta=5.7^{\circ}$.

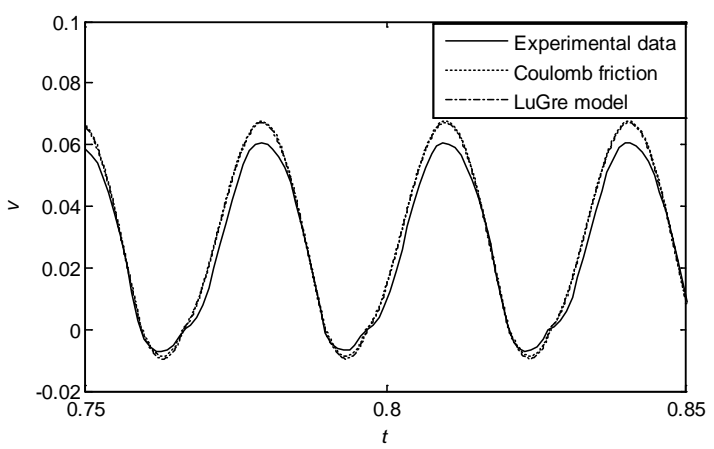

Figure 10. The time history of the dimension velocity $v$ resulting from numerical simulation of Coulomb friction in dotted curve and LuGre model in dashed curve of the exact equation of the motion and the experimental data in solid curve.

The approximate average velocity of the experimental data, the numerical simulation with the Coulomb friction and the LuGre model is $0.0254 \mathrm{~m} / \mathrm{s}, 0.0287 \mathrm{~m} / \mathrm{s}, 0.0281 \mathrm{~m} / \mathrm{s}$, respectively. Through numerical simulation, we can draw the conclusion that, when the motion of the whole system belongs to the fourth type, then the simulation results of the Coulomb friction and the LuGre model are nearly the same. That is to say, it is hard to distinguish the vibration-driven system with the Coulomb friction and the friction of LuGre model from the nonstick-slip motion.

\section{The Dynamical Behavior of the System with the LuGre Model}

In section 4, we just discuss the four types of movement forms of the single module vibration-driven system. The physical parameters values in the system are selected according to the experiment measuring. In fact, because of the lack of measuring methods and measuring tools, the measuring results may have some errors.

Next, we will study what will happen of the dynamical behaviors under different parameter values.

The equation motion of the vibration-driven system with the LuGre model is

$$
\begin{aligned}
& \text { if } \dot{x}>0, \\
& \dot{x}=v, \\
& \dot{v}=A \omega^{2} \sin (\omega t)-\frac{\sigma_{0} z}{M+m}-\frac{\left(\sigma_{1}+\sigma_{2}\right) v}{M+m}+\frac{\sigma_{0} \sigma_{1} v z}{(M+m)^{2} g\left(\mu_{+}+\left(f_{+}-\mu_{+}\right) e^{\left.-\left(\frac{v}{v_{s}}\right)^{2}\right)}\right.} \\
& \dot{z}=v-\frac{\sigma_{0} v z}{(M+m) g\left(\mu_{+}+\left(f_{+}-\mu_{+}\right) e^{\left.-\left(\frac{v}{v_{s}}\right)^{2}\right)}\right.} .
\end{aligned}
$$




$$
\begin{aligned}
& \text { if } \dot{x}<0 \\
& \left\{\begin{array}{l}
\dot{x}=v \\
\dot{v}=A \mu \omega^{2} \sin (\omega t)-\frac{\sigma_{0} z}{M+m}-\frac{\left(\sigma_{1}+\sigma_{2}\right) v}{M+m}-\frac{\sigma_{0} \sigma_{1} v z}{(M+m)^{2} g\left(\mu_{-}+\left(f_{-}-\mu_{-}\right) e^{-\left(\frac{v}{v_{s}}\right)^{2}}\right)} \\
\dot{z}=v+\frac{\sigma_{0} v z}{(M+m) g\left(\mu_{-}+\left(f_{-}-\mu_{-}\right) e^{-\left(\frac{v}{v_{s}}\right)^{2}}\right)} .
\end{array}\right.
\end{aligned}
$$

Let $k_{1}=\frac{f_{+}}{\mu_{+}} \quad, \quad k_{2}=\frac{f_{-}}{\mu_{-}} \quad, \quad k_{3}=\frac{\mu_{-}}{\mu_{+}} \quad, \quad \mathrm{a}=\frac{\sigma_{0}}{\omega^{2}(M+m)} \quad, \quad b=\frac{\sigma_{1}}{\omega(M+m)} \quad, \quad c=\frac{\sigma_{2}}{\omega(M+m)}$, $d=\frac{A \sigma_{0}}{\mu_{+}(M+m) g}$. And assume that $x^{*}=\frac{x}{A}, v^{*}=\frac{v}{A \omega}, z^{*}=\frac{z}{A}, v_{s}^{*}=\frac{v_{s}}{A \omega}, t^{*}=\omega t$. For the sake of brevity, we omit the asterisks. Then the above equations can be rewritten as

$$
\begin{aligned}
& \text { if } v>0, \\
& \left\{\begin{array}{l}
\dot{x}=v \\
\dot{y}=\mu \sin t-a z-(b+c) v+\frac{b d v z}{1+\left(k_{1}-1\right) e^{-\left(\frac{v}{v_{s}}\right)^{2}},} \\
\dot{z}=v-\frac{d v z}{1+\left(k_{1}-1\right) e^{-\left(\frac{v}{v_{s}}\right)^{2}}}
\end{array}\right. \\
& \left\{\begin{array}{l}
\dot{x}=v \\
\dot{v}=\mu \sin t-a z-(b+c) v-\frac{k_{3}}{\left(1+\left(k_{2}-1\right) e^{-\left(\frac{v}{v_{s}}\right)^{2}}\right.} . \\
\left.\dot{z}=v+\frac{k_{3}\left(1+\left(k_{2}-1\right) e^{-\left(\frac{v}{v_{s}}\right)^{2}}\right.}{(d v z}\right)
\end{array}\right.
\end{aligned}
$$

By substituting equation (4.1) into the definition of $k_{1}, k_{2}$ and $k_{3}$ and after some simplifying, we have

$$
k_{1}=\frac{f_{0}-\tan \theta}{\mu_{0}-\tan \theta}, k_{2}=\frac{f_{0}+\tan \theta}{\mu_{0}+\tan \theta}, k_{3}=\frac{f_{0}+\tan \theta}{\mu_{0}-\tan \theta}
$$

Due to the physical meanings of the friction coefficients, we may know that $k_{1}>1, k_{2}>1, k_{3}>1$. All the parameters in the LuGre models are given in section 4 .

Now we will discuss the effects of these parameters on the vibration-driven system with the LuGre model. Due to space limitations in this paper, we just discuss the magnitude of the excitation and the friction. 
Next, they will be analyzed one by one.

\subsection{The Effect of the Excitation Amplitude $A$}

In the following discussion, the values of other parameters will be unchanged without pointing out, except for the excitation amplitude $A$. The parameter values of the LuGre model are taken as

$$
\sigma_{0}=4 \times 10^{5} \mathrm{~N} / \mathrm{m}, \sigma_{1}=100 \mathrm{Ns} / \mathrm{m}, \sigma_{2}=0.04 \mathrm{Ns} / \mathrm{m}, v_{s}=0.002 \mathrm{~m} / \mathrm{s}
$$

For the physical parameters, we just talk about the excitation amplitude $A$, the excitation frequency $\omega$ and the friction coefficients ratio $k_{1}, k_{2}, k_{3}$. In fact, these three dimensionless parameters are just controlled by the static friction coefficient $f_{0}$, dynamic friction coefficient $\mu_{0}$ and the angle of inclination $\theta$ as given in(4.1).

Firstly, we will discuss the effect of the excitation amplitude $A$ when the other parameters remain unchanged. We need note that the velocity of the dimensional equation is $A \omega$ times of the dimensionless one. And the new period of the dimensionless system is $2 \pi$.

We take the other parameters as $\omega=65.4 \pi, \mu_{0}=0.18, f_{0}=0.21, \theta=1.7^{\circ}$. Through numerical simulation, it is found that the velocity of the rigid body can display different dynamic behaviors by changing the magnitude of the excitation amplitude $A$. Moreover, it can predict the system behavior whereas the experiment can't do, as the shortcomings of the experimental equipments and measuring methods. Fig.10 shows that under the low excitation amplitude, the dimensionless velocity meet simple harmonic vibration. By subtracting the rigid displacements, we obtain the phase portrait of the rigid body. The phase portrait shows that the steady state velocity of the rigid body is monocyclic.
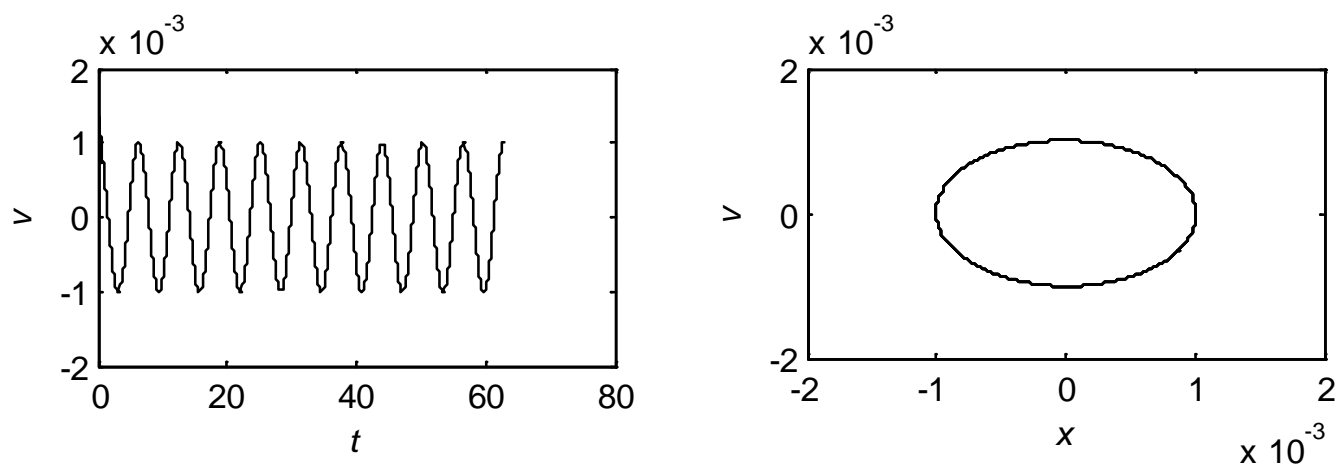

Figure 11. The time history of the velocity and phase portrait, where $A=10^{-6} \mathrm{~m}$.

As the increase of the excitation amplitude $A$, the system can illustrate different kinds of movement forms which have a good agreement with the experimental data. The following figures give the evidences.
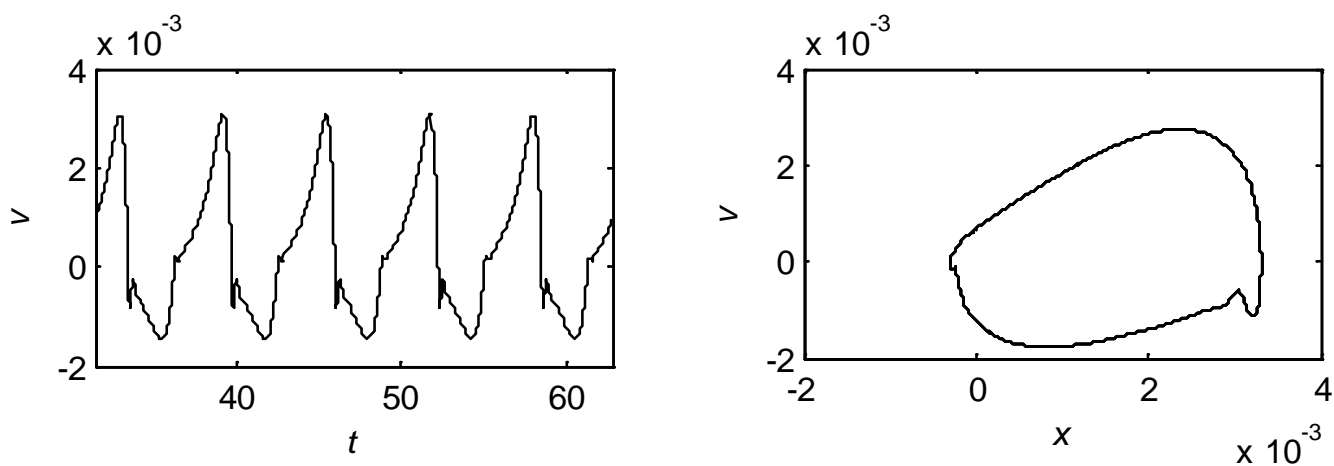

Figure 12. The time history of the velocity and phase portrait, where $A=3 \times 10^{-4} \mathrm{~m}$. 

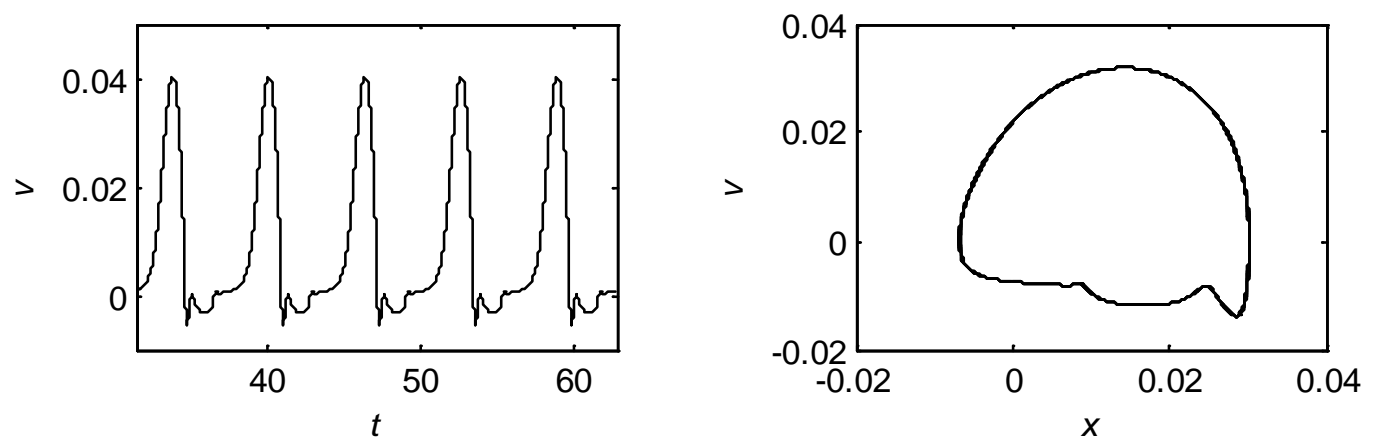

Figure 13. The time history of the velocity and phase portrait, where $A=4 \times 10^{-4} \mathrm{~m}$.
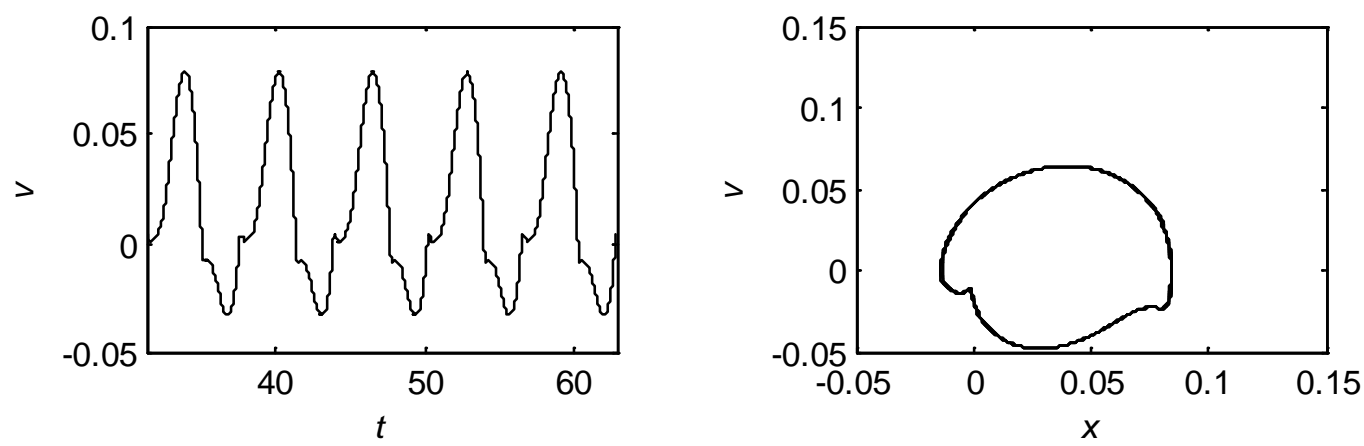

Figure 14. The time history of the velocity and phase portrait, where $A=5 \times 10^{-4} \mathrm{~m}$.
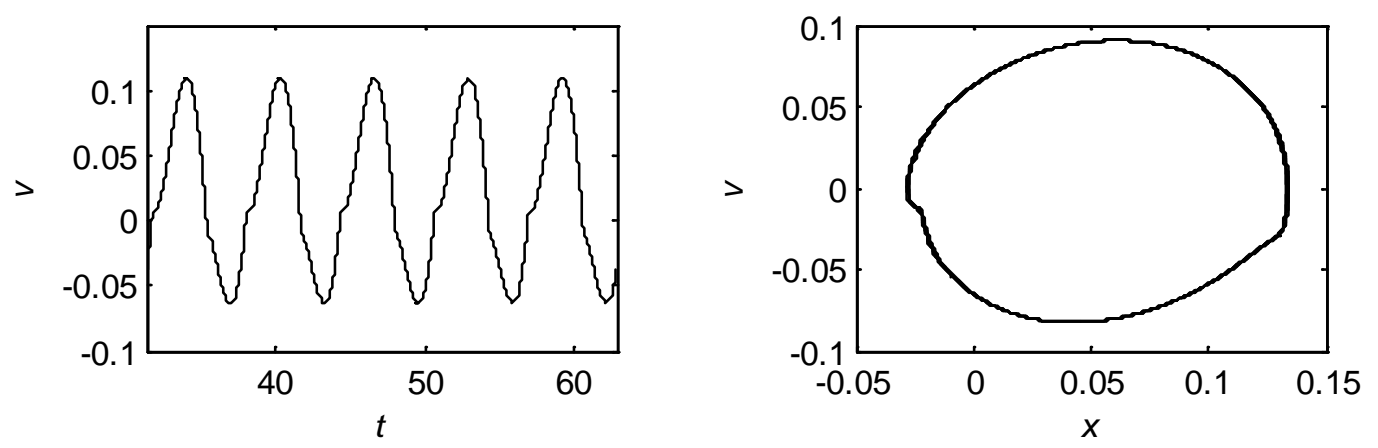

Figure 15. The time history of the velocity and phase portrait, where $A=6 \times 10^{-4} \mathrm{~m}$.
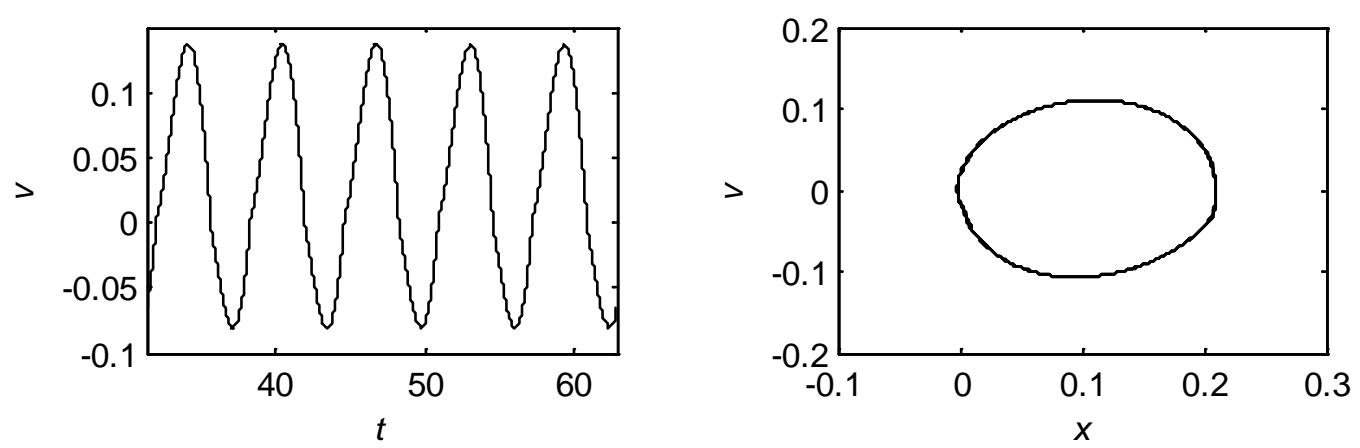

Figure 16. The time history of the velocity and phase portrait, where $A=8 \times 10^{-4} \mathrm{~m}$. 
Obviously, by variation the excitation amplitude $A$, one can simulate all the four movement forms that found in the experiment. Furthermore, the phase portrait of the system is always periodic.

\subsection{The Effect of the Excitation frequency $\omega$}

The excitation frequency has the same effect as the excitation amplitude $A$. The following figures illustrate the system's motion as the excitation frequency changes.
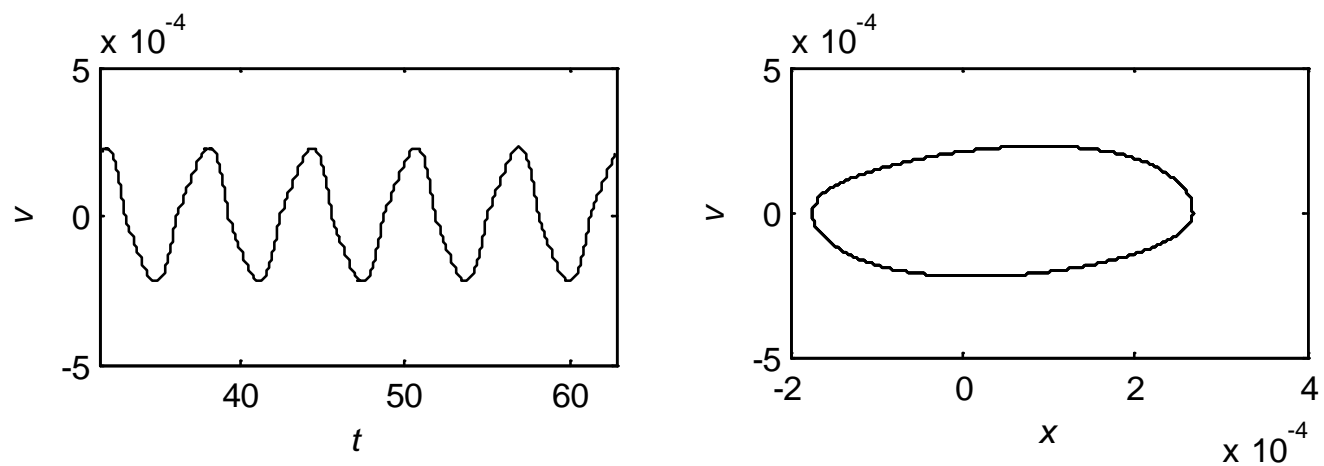

Figure 17. The time history of the velocity and phase portrait, where $\omega=30 \pi s^{-1}$.
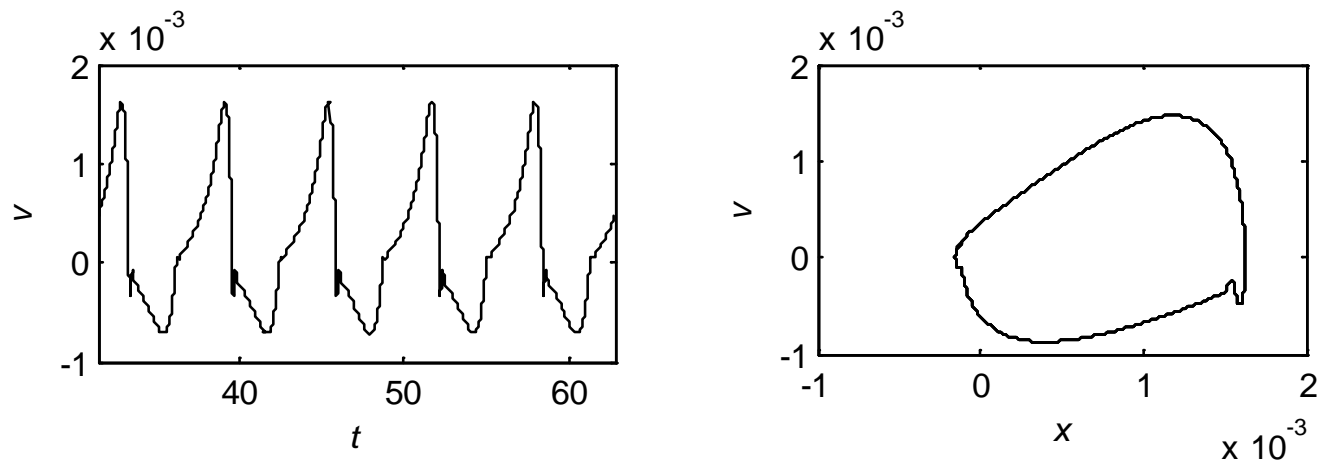

Figure 18. The time history of the velocity and phase portrait, where $\omega=46 \pi s^{-1}$.
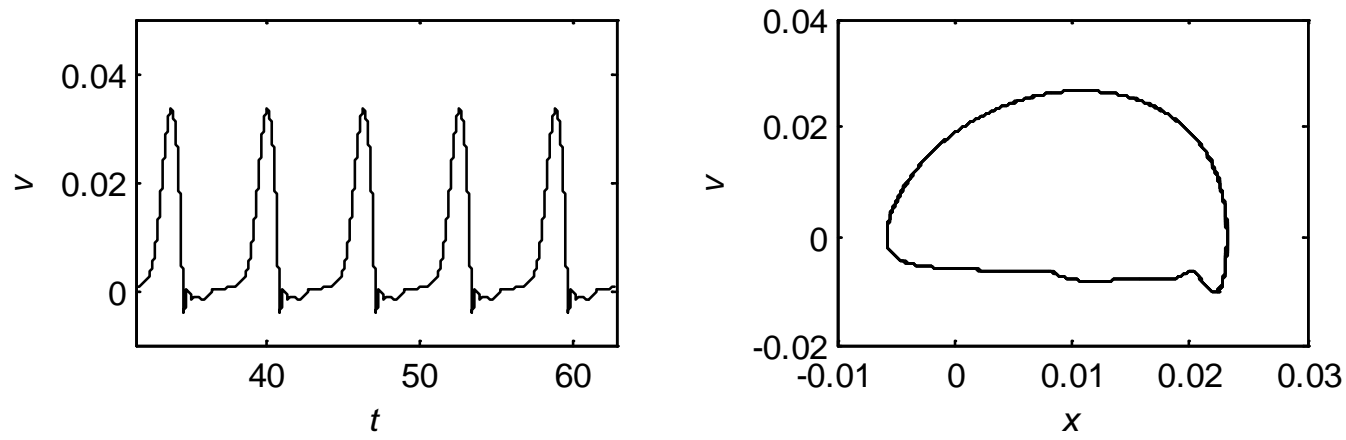

Figure 19. The time history of the velocity and phase portrait, where $\omega=52 \pi s^{-1}$. 

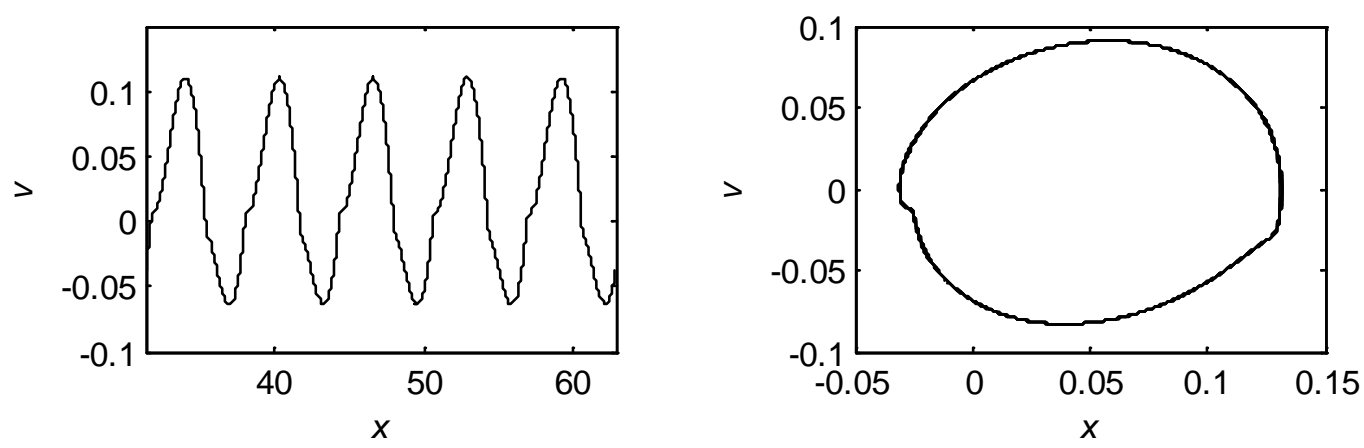

Figure 20. The time history of the velocity and phase portrait, where $\omega=65 \pi \mathrm{s}^{-1}$.
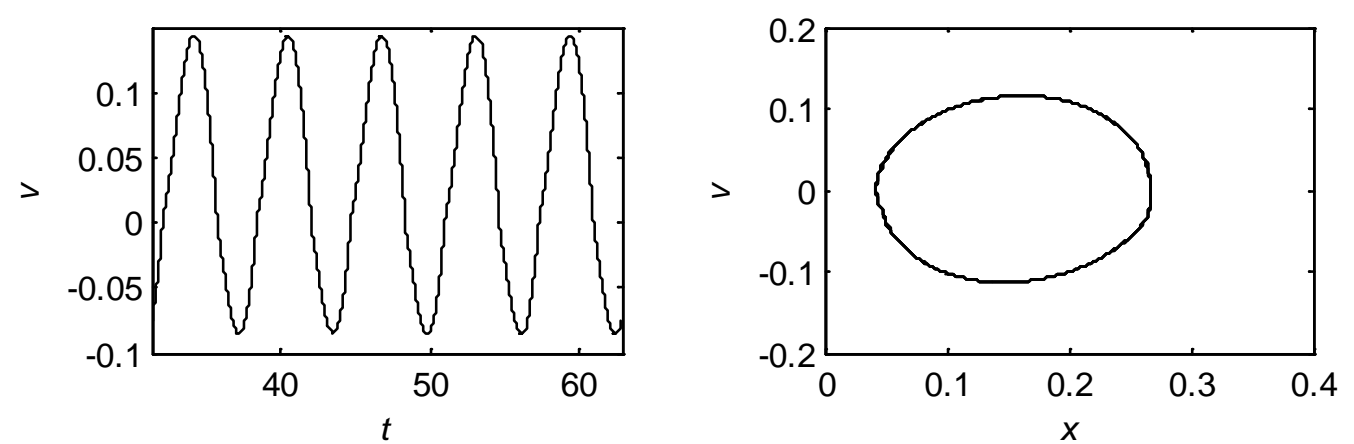

Figure 21. The time history of the velocity and phase portrait, where $\omega=80 \pi s^{-1}$.

This part can be analyzed as the above part. One should notice that the excitation frequency in the experiment has limits. The frequency of excitation must be below $5000 \mathrm{HZ}$.

In conclusion, by varying the amplitude and frequency of the excitation, one can obtain all the four types of movement forms as the experimental data.

\subsection{The Effect of the Dynamic Friction Coefficient $\mu_{0}$}

The vibration-driven system can move due to the internal mass interaction with the rigid body and the anisotropic friction force. That is to say, friction force plays a crucial role in the vibration-driven system.

As the friction coefficient is non-negative, i.e., $\mu_{-}>\mu_{+} \geq 0$, thus, from equation (4.1), one can deduce that $\mu_{0} \geq \tan \theta=0.03$. Moreover, the dynamic friction coefficient $\mu_{0}$ is no more than the static friction coefficient $f_{0}$ as the common sense. Then, the range of the dynamic friction coefficient is $0.03 \leq \mu_{0} \leq 0.21$.
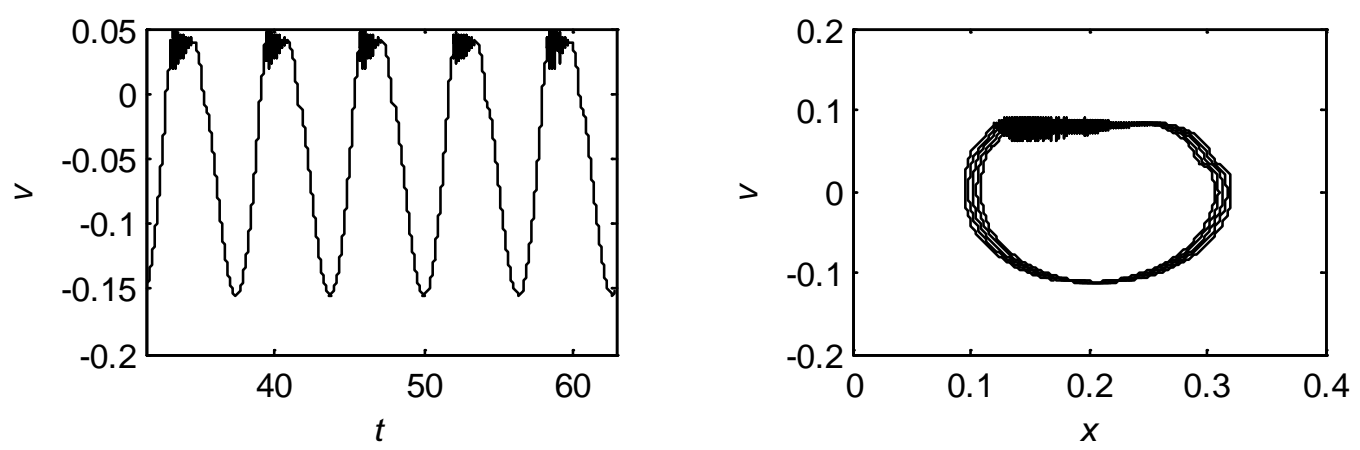

Figure 22. The time history of the velocity and phase portrait, where $\mu_{0}=0.03$. 

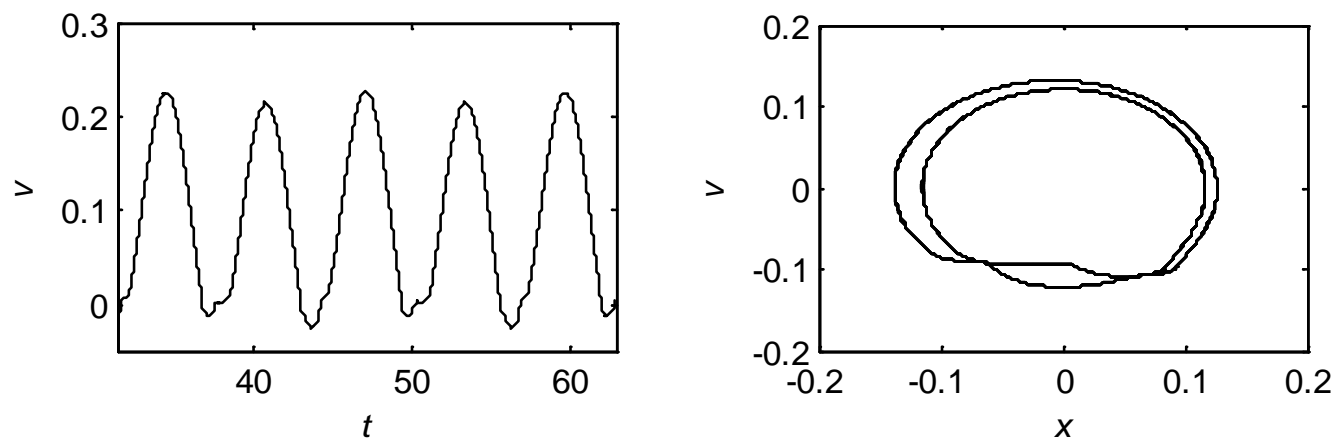

Figure 23. The time history of the velocity and phase portrait, where $\mu_{0}=0.04$.
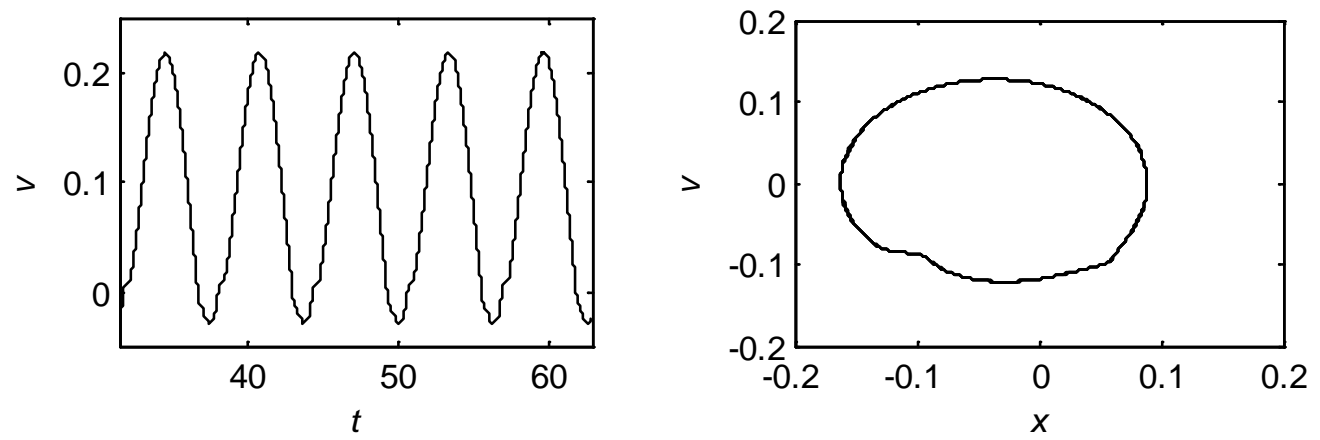

Figure 24. The time history of the velocity and phase portrait, where $\mu_{0}=0.05$.
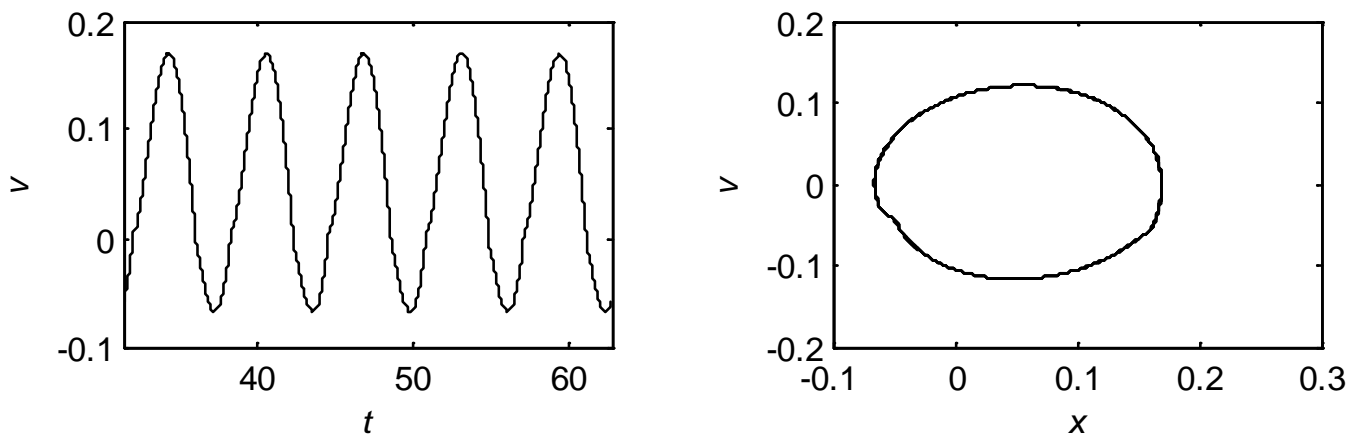

Figure 25. The time history of the velocity and phase portrait, where $\mu_{0}=0.10$.
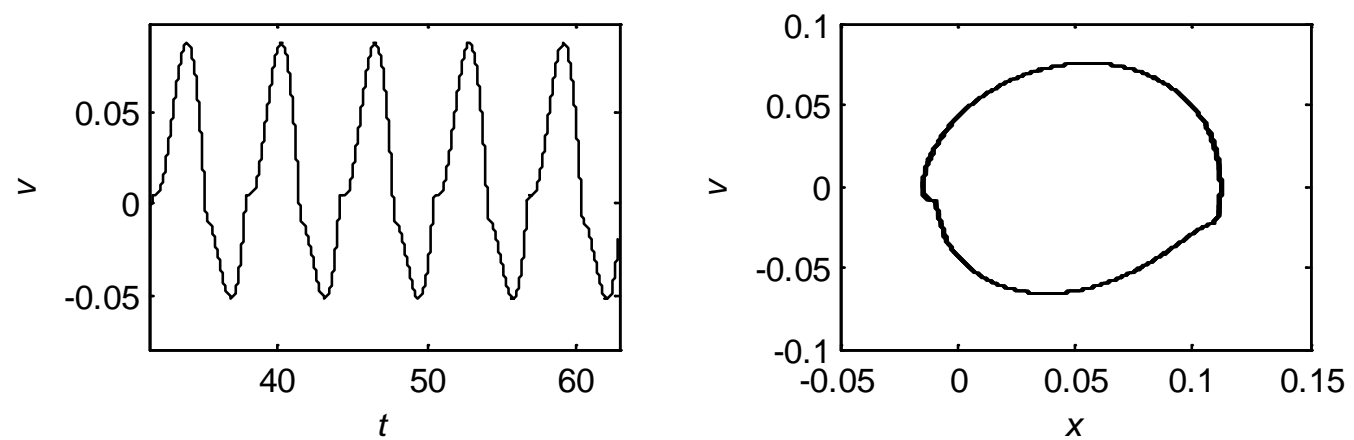

Figure 26. The time history of the velocity and phase portrait, where $\mu_{0}=0.21$.

It's obvious that, see Fig. 22, when the dy namic friction coefficient is $\mu_{0}=0.03$, the rigid body does a quasi-period motion. We should also pay attention to the situation of $\mu_{0}=0.04$. Then the motion of body 
$M$ displays the dynamic behavior of period two shown in Fig. 23.

Different from the above two parts, when the dynamic friction coefficient $\mu_{0}$ is smaller enough than the static friction coefficient $f_{0}$, then the system shows some strange dynamic behaviors, such as quasi-period motion and period two motion. From Fig.24-Fig.26, for the larger dynamic friction coefficient, no matter how we change its value, the vibration-driven system just displays the third and fourth type movement forms. In the simulation, no first and second type movement forms appear.

\subsection{The Effect of the Static Friction Coefficient $f_{0}$}

In this part, we just talk about the effect of the static fiction coefficient $f_{0}$ on the dy namic behavior of the vibration-driven system. In the other authors' researches, they didn't consider the static friction. They regarded the dynamic friction coefficient as the static friction coefficient. Actually, the static friction plays an important role in the vibration-driven system. We all know that the dynamic friction is not more than the static friction, i.e., $\mu_{0} \leq f_{0}$. That is to say, the range of the static friction coefficient is $0.18 \leq f_{0} \leq 1$. The Figs.27-30 show under different static friction coefficients, the vibration-driven system can illustrate different dynamic behaviors.
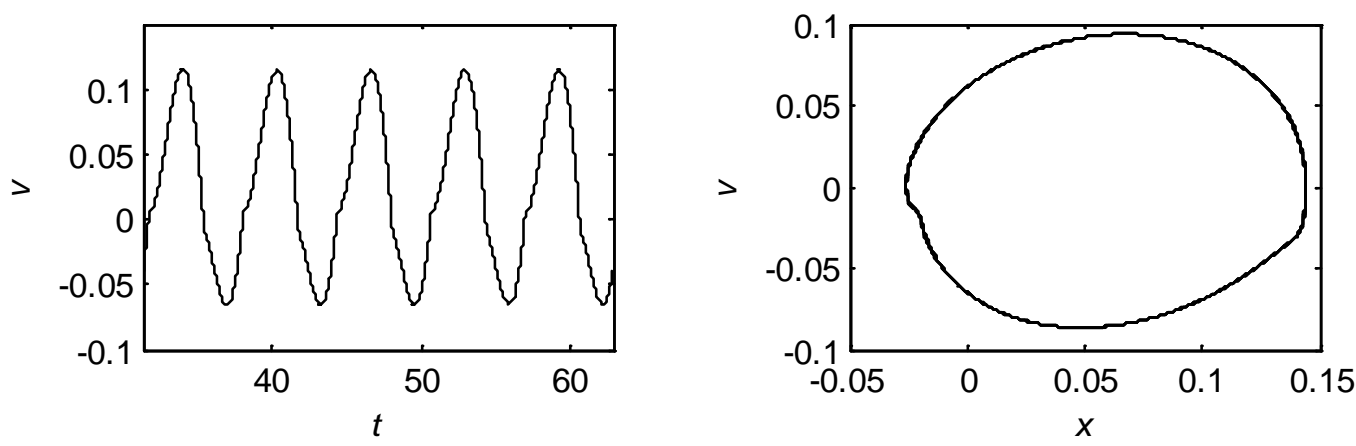

Figure 27. The time history of the velocity and phase portrait, where $f_{0}=0.18$.
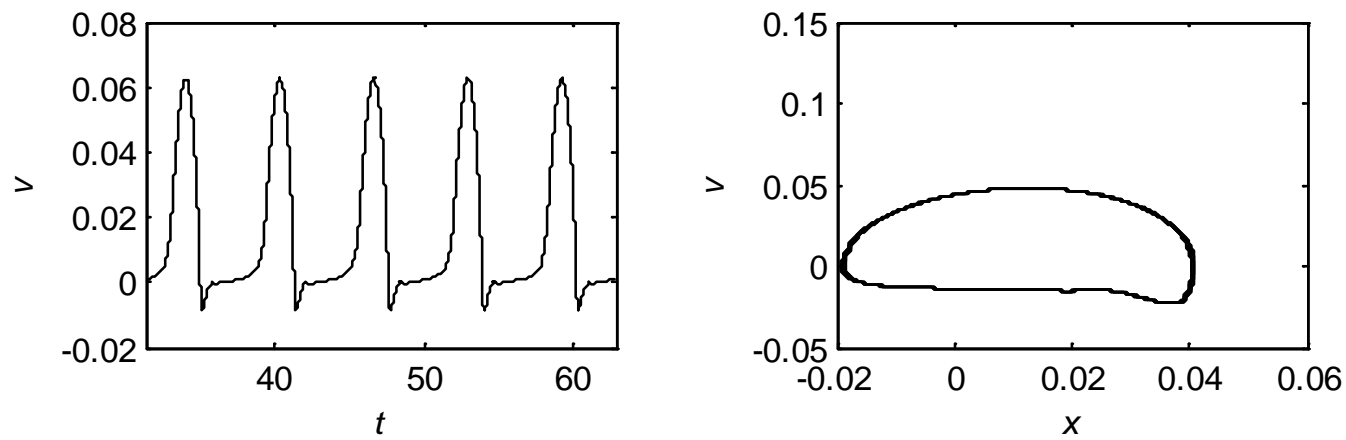

Figure 28. The time history of the velocity and phase portrait, where $f_{0}=0.35$. 

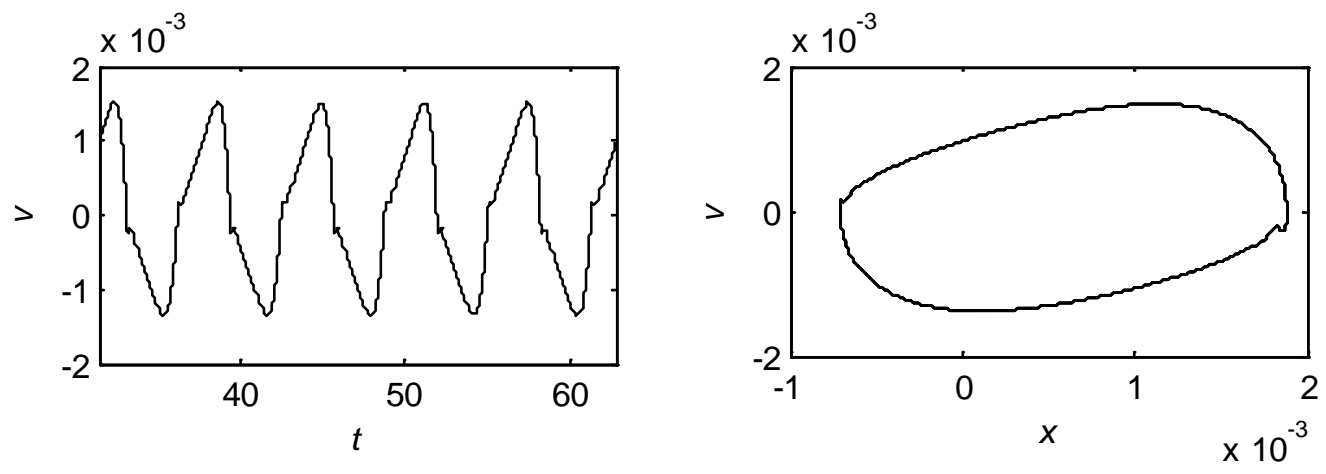

Figure 29. The time history of the velocity and phase portrait, where $f_{0}=0.5$.
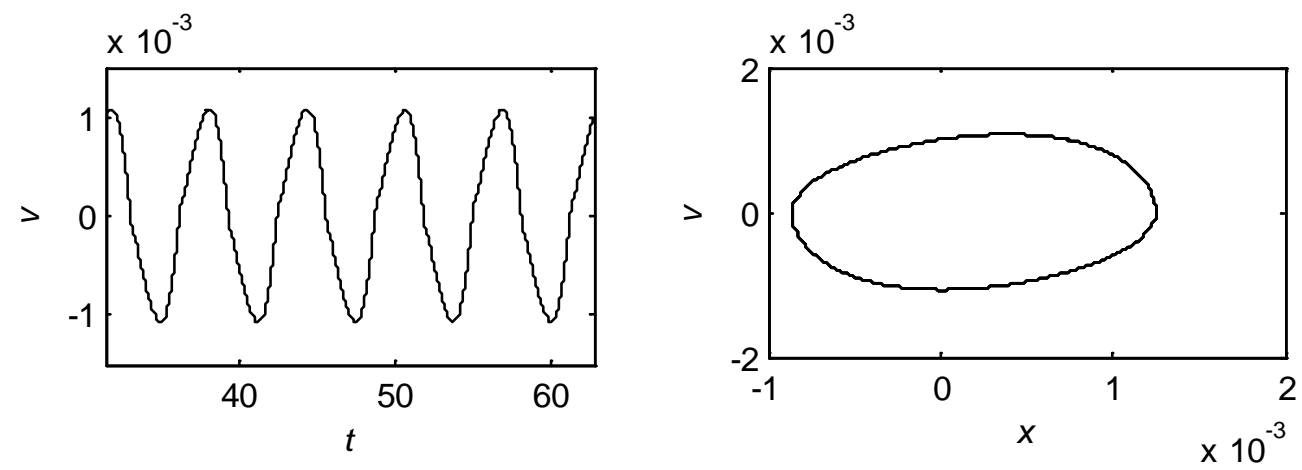

Figure 30. The time history of the velocity and phase portrait, where $f_{0}=1$.

From these figures, one can find that, the dimensionless velocity $v$ changes with the static friction coefficient $f_{0}$. Moreover, through adjusting the static friction coefficient, one can obtain all the four types movement forms.

\section{Summary and Conclusion}

In this paper mathematical modeling of the single module vibration-driven system with Coulomb friction and LuGre model is shown, respectively. Numerical analysis of the system was carried out for a set of system parameters and physical parameters. There are four types of movement forms of the rigid body from the experimental results, i.e., three type of stick-slip motion and one type of nonstick-slip motion.

We do an experiment to find out a better friction model which is a better agreement with the experiment data. From the view point of quantitative and qualitative, it is found that the LuGre model is a better friction model after doing numerical simulations.

Then one applies the LuGre model to predict other dynamic behaviors of the single module vibration-driven system. Under different physical parameters, the system shows different movement forms. In this paper, we just talk about the excitation amplitude, the excitation frequency, the static and dynamic friction coefficients. However, for lack of space, we omit the study of the other parameters.

Through numerical simulations, one can find other dynamical behaviors of the system which can't be observed in the experiment. For example, Fig. 22 and Fig. 23 display new kinds of movement forms which are different from the known four types. By changing the magnitude of the amplitude and frequency of the excitation and static friction coefficient, one can obtain all the four types movement forms, except the dynamic friction coefficient. Moreover, if the dynamic friction coefficient is much smaller than the static one, the system will display quasi-periodic and periodic two motions.

Acknowledgments

This research was supported by the National Nature Science Foundation of China under grant number 


\section{References}

1. F. L. Chernous.Ko, "On the motion of a body containing a movable internal mass," Doklady Physics, vol. 50, no. 11, pp. 593-597, 2005.

2. N. N. Bolotnik, N.N., T. Y. Figurina and F. L. Chernous Ko," Optimal control of the rectilinear motion of a two-body system in a resistive medium," Journal of Applied Mathematics and Mechanics, vol. 76, no. 1, pp. 1-14, 2012.

3. N. N. Bolotnik, et al., "Dynamics of controlled motion of vibration-driven systems," Journal of Computer and Systems Sciences International, vol. 45, no. 5, pp. 831-840, 2006.

4. N. N. Bolotnik, and T. Y. Figurina, "Optimal control of periodic motions of vibration-driven systems," IFAC Proceedings, vol. 40, no. 14, pp. 142-147, 2007.

5. F. L. Chernous Ko, "Analysis and optimization of the motion of a body controlled by means of a movable internal mass," Journal of Applied Mathematics and Mechanics, vol. 70, no. 6, pp. 819-842, 2006.

6. A. G. Egorov, and O. S. Zakharova, "The optimal quasi-stationary motion of a vibration-driven robot in a viscous medium," Russian Mathematics, vol. 56, no. 2, pp. 50-55, 2012.

7. H .B. Fang, and J. Xu, "Dynamic analysis and optimization of a three-phase control mode of a mobile system with an internal mass," Journal of vibration and control, vol. 17, no. 1, pp. 19-26, 2011.

8. H. B. Fang, and J. Xu, "Stick-slip effect in a vibration-driven system with dry friction: sliding bifurcations and optimization," Journal of Applied Mechanics, vol. 81, no. 5, pp. 1-10, 2014.

9. H. B. Fang, and J. Xu, "Dynamics of a mobile system with an internal acceleration-controlled mass in a resistive medium," Journal of sound and vibration, vol. 330, no. 16, pp. 4002-4018, 2011.

10. C.H.Y. Hu, C.E. Kreuzerand, and F. L. Chernousko, "Dynamics of a body controlled by internal motions," in Iutam Symposium on Dynamics and Control of Nonlinear Systems with Uncertainty: Proceedings of the IUTAM Symposium held in Nanjing, China, pp. 227-236, 2006.

11. A. G. Yegorov, and O. S. Zakharova, "The energy-optimal motion of a vibration-driven robot in a resistive medium," Journal of Applied Mathematics and Mechanics, vol. 74, no. 4, pp. 443-451, 2010.

12. H.B. Fang, and J. Xu, "Controlled motion of a two-module vibration-driven system induced by internal acceleration-controlled masses," Archive of Applied Mechanics, vol. 82, no. 4, pp. 461-477, 2012.

13. K. Zimmermann, et al., "Dynamics of a two-module vibration-driven system moving along a rough horizontal plane," Multibody System Dynamics, vol. 22, no. 2, pp. 199-219, 2009.

14. H.B. Fang, and J. Xu, "Dynamics of a three-module vibration-driven system with non-symmetric Coulomb's dry friction," Multibody System Dynamics, vol. 27, no. 4, pp. 455-485, 2012.

15. K. Zimmermann, et al. "An approach to the modelling of worm-like motion systems with finite degree of freedomfirst steps in technical realization," in Proceedings of the Fourth International Conference on Climbing and Walking Robots, Karlsruhe, pp. 561-568, 2001.

16. R.A. Ibrahim, "Friction-induced vibration, chatter, squeal, and chaos-Part II: dynamics and modeling," Applied Mechanics Reviews, vol. 47, no. 7, pp. 227-253, 1994.

17. Jedynak R., M. Sulek, "Numerical and experimental investigation of plastic interaction between rough surfaces, " Arabian Journal foe science and engineering, vol. 30, pp. 4165-4177, 2014.

18. L.L. Liu,et al, "An overview of friction models in mechanical systems," Advances in Mechanics, vol. 8, no. 2, pp. 201-213, 2008.

19. Q. Ding, H.M. Zhai, "The advance in researches of friction dynamics in mechanical system," vol. 43, no. 1, pp. 112-131, 2013.

20. Pennestrì E., Valerio Rosssi, Salvini P, Valentini P., "Review and comparison of dry friction force models," Nonlinear dynamics, vol. 83, pp. 1785-1801, 2016.

21. J. Swervers, et al., "An integrated friction model structure with improved presliding behavior for accurate friction compensation," IEEE Transactions on Automatic Control, vol. 45, no. 4, pp. 675-686, 2000.

22. K. Johanastrom, C. Canudas de Wit, "Revisiting the LuGre friction model," IEEE Control Systems, vol. 28, no. 6, pp. 101-114, 2008

23. N. Barahanov, R. Ortega, "Necessary and sufficient conditions for passivity of the LuGre friction model," IEEE Transactions on Automatic Control, vol. 45, no. 4, pp. 830-832, 2000. 
24. Y. Wang, Z.He and G.X. Wang, "A practical friction model," vol. 15, no. 8, pp. 59-63, 2011.

25. J. Awrejcewicz, D. Grzelczyk and Y. Pyryev. "On the stick-slip vibrations continuous friction model," in Proceedings of the 9th Conference on Dynamical Systems-Theory and Applications, Poland, pp. 113-120, 2007.

26. A.Saha, et al., "A modified LuGre friction model for an accurate prediction of friction force in the pure sliding regime," International Journal of Non-Linear Mechanics, vol. 80, pp. 122-131, 2016.

27. A.Wijata, et al., "Mathematical model for two-dimensional dry friction modified by dither," Mathematics and Mechanics of Solids, pp. 1-14, 2016.

28. N.N. Bolotnik, F.L. Chernousko and T.Y. Figurina, "Optimal control of a two-body system moving in a viscous medium," IFAC Proceedings, vol. 43, no. 14, pp. 1308-1313, 2010.

29. F.L. Chernous Ko., "The optimal periodic motions of a two-mass system in a resistant medium," Journal of Applied Mathematics and Mechanics, vol. 72, no. 2, pp. 116-125, 2008.

30. N.N. .Bolotnik, and T.Y. Figurina, "Optimal control of the rectilinear motion of a rigid body on a rough plane by means of the motion of two internal masses," Journal of Applied Mathematics and Mechanics, vol. 72, no. 2, pp. 126-135, 2008.

31. F.L. Chernous'Ko, "The optimum rectilinear motion of a two-mass system," Journal of Applied Mathematics and Mechanics, vol. 66, no. 1, pp. 1-7, 2002.

32. A. Preumont, Vibration Control of Active Structures, Springer, 2011. 